\title{
On the effect of topography on surface wave propagation in the ambient noise frequency range
}

\author{
Andreas Köhler · Christian Weidle · Valérie Maupin
}

Received: date / Accepted: date

\begin{abstract}
Due to the increasing popularity of analyzing empirical Green's functions obtained from ambient seismic noise, more and more regional tomographical studies based on shortperiods surface waves are published. Results could potentially be biased in mountainous regions where topography is not small compared to the wavelength and penetration depth of the considered waves. We investigate the effect of topography on the propagation of short-period Rayleigh waves empirically by means of synthetic data using a spectral element code and a 3-D model with real topography. We show that topography along a profile through the studied area can result in an underestimation of phase velocities of up to about $0.7 \%$ at the shortest investigated period ( 3 seconds). Contrary to the expectation that this bias results from the increased surface distance along topography, we find that this error can be estimated by local topographic contrasts in the vicinity of the receiver alone. We discuss and generalize our results by considering topographic profiles through other mountain ranges and find that southern Norway is a good proxy to assess the topography effect. Nevertheless, topographic bias on phase velocity measurements is in general not large enough to significantly affect recovered velocity variations in the ambient noise frequency range.
\end{abstract}

Keywords Surface Waves · Topography · Wave Propagation · Phase Velocity

\footnotetext{
A. Köhler, C. Weidle*, V. Maupin

Department of Geosciences, University of Oslo

Post Box 1047, 0316 Oslo, Norway

Tel.: +47-22856678

Fax: +47-22854215

E-mail: andreas.kohler@geo.uio.no

* now at: Dept. of Geosciences, Christian-Albrechts-Universitt zu Kiel, Otto-Hahn-Platz 1, 24118 Kiel, Germany
}

\section{Introduction}

Motivation for this study are findings of a surface wave tomography of southern Norway based on ambient seismic noise analysis (Köhler et al, 2011). Low velocity anomalies of about 1-3\% have been found between 3 and 10 seconds period within the western part of southern Norway, a region characterized by large topographic contrasts. In this region, elevation reaches $2000 \mathrm{~m}$ a.s.l. and high mountains are cut by fjords with up to 1000 water depth, summing to vertical contrasts of up to $3 \mathrm{~km}$ on relatively short horizontal distances (Figure 1). Due to the rather small penetration depths and short wavelengths of ambient seismic noise (about $9 \mathrm{~km}$ at 3 seconds), we have to take into consideration that topography may affect the waves dispersion and the inferred velocity from the traveltime measurement. If so, we need to correct for this effect before interpreting the results in terms of crustal structure.

The wave (phase- or group-) velocity between two points is generally evaluated by dividing the interstation distance by an estimate of the traveltime difference (or phase difference). Great attention is usually devoted to the measurement of the time difference but we seldom question the precision of the distance used in the evaluation. For surface waves, the distance is taken as the distance along the surface of a spherical or elliptical Earth model, implicitly assuming that surface waves travel perfectly horizontally and do not follow the topography. This approximation is likely to be true for long period waves which have their maximum of energy at depth much greater than the amplitude of topographic variations. For smaller periods, and especially for the Rayleigh wave fundamental mode whose propagation is strongly controlled by the presence of the free surface, one may imagine that short period waves follow the surface and thereby travel a distance larger than the direct path. Failure to use the right distance in the evaluation of the velocity will lead to biases 
towards too slow velocities. A simplistic approach to evaluate the possible effect of topography on the phase velocity between two points at the Earth's surface is therefore just to compare the difference in distance between the two points using a purely horizontal path $\left(d_{\infty}\right)$ or following the topography $\left(d_{\lambda}\right.$, filtered at a given wavelength $\left.\lambda\right)$. In our study area in southern Norway, using a topographic profile lowpass filtered at $2 \mathrm{~km}$, this simple exercise indicates that the ratio $\frac{d_{\infty}}{d_{2}} \approx 0.97$ and the measured phase velocities may be underestimated by up to $3 \%$.

Most studies on the relation between surface wave propagation and topography emphasize the scattering effect and the associated attenuation, in particular for $\mathrm{Lg}$ waves (Fu and $\mathrm{Wu}, 2001$; $\mathrm{Wu}$ and $\mathrm{Wu}, 2001$; Fu et al, 2002). Impact of topography and scattering on phase velocity measurements of surface waves has been studied for period bands longer than 10 seconds by Snieder (1986). Using very smooth topography and the Born approximation, the author showed that a topography of $1 \mathrm{~km}$ (for example) introduced a maximum error of $0.5 \%$ in the phase velocity estimate. The phase velocity perturbations in his model are largest at 20 seconds and decrease towards shorter and longer periods. The velocity variation is mainly related to the thickening of the crustal waveguide associated with the topography and not the topography itself, and is therefore not the mechanism which is important for our purpose.

The effect of a corrugated surface on the propagation of Rayleigh waves in a homogeneous substratum has been studied analytically by Maradudin and coworkers in several papers (e.g., Eguiluz and Maradudin, 1983; Huang and Maradudin, 1987; Mayer et al, 1991; Maradudin et al, 1991). They show that in addition to the expected attenuation, the relief of the free surface induces a reduction in the phase velocity (Eguiluz and Maradudin, 1983; Huang and Maradudin, 1987) and emergence of both Love waves and higher Rayleigh modes (Mayer et al, 1991; Maradudin et al, 1991). Since emergence of these modes is not expected in a homogeneous halfspace and occurs only at large wavelengths compared to the wavelength of the relief, these authors argue that these waves sense the uppermost part of the model between peaks and troughs as an upper layer (that is only partially filled with material) with reduced effective velocity. This explains thus that topographic relief reduces the velocity of waves at long wavelength. It does not explain however that, after decreasing close to the relief characteristic wavelength, their predicted velocity reduction increases again strongly for waves with shorter wavelength for which Love waves do not emerge anymore. In this wavelength range, the distance factor discussed above may play a large role. For a scaleinvariant, fractal topography however, the apparent low velocity upper layer may be important in both wavelength ranges.

The velocity reduction calculated by Huang and Maradudin (1987) assumes that the spectrum of the topography is Gaus- sian, which is not the case for the Earth. It is therefore difficult to rely solely on these results to evaluate the bias that we can expect, but we will later compare our results with what can be inferred from their studies.

In the present study, we investigate the effect of topography based on synthetic data generated in a homogeneous halfspace model with a corrugated free surface that reflects the real topography from the western part of southern Norway. A homogeneous halfspace model is a good approximation for our study region as previous studies indicate a rather homogeneous crustal structure (Stratford et al, 2009; Köhler et al, 2011). We can then single out the effect of the presence of topography itself on Rayleigh wave fundamental mode phase velocity at periods shorter than 10 seconds. The results for southern Norway are then generalized to evaluate the velocity biases that can be expected for other mountains ranges.

\section{Topography in southern Norway}

The topography of southern Norway is often referred to as dome-like with elevated planar surfaces above $1000 \mathrm{~m}$ and peak elevations above $2000 \mathrm{~m}$. While the topography falls off rather gently eastwards from the center, the falloff towards the coastal regions, particularly in the western part of southern Norway is generally steeper and high topography is incised by deep fjords (Lidmar-Bergström et al, 2000). This leads to locally large topographic contrasts, as illustrated by the profile that we chose for our analysis (Figure 1). This profile shows by far the highest topographic contrasts in elevation along interstation profiles of the MAGNUS network, a temporary network from which data was used in the seismic noise study of Southern Norway (Weidle et al, 2010; Köhler et al, 2011). We use topographic data from the global 30-arc-second gridded digital elevation model GLOBE (GLOBETaskTeam et al, 1999) which has a sampling rate of $1 \mathrm{~km}$ in our model. The largest contrast in altitude along our profile occurs in the Sognefjord region, at a distance of about $80 \mathrm{~km}$ from the coast, where the free surface (without water) varies in altitude by $2400 \mathrm{~m}$ over a horizontal distance of $9 \mathrm{~km}$ - an average slope of $27 \%$.

\section{Generation of synthetic data}

It is now well established that the cross-correlation of noise seismograms yields the surface wave Green's function between two receivers (Shapiro and Campillo, 2004; Sabra et al, 2005). This has also been shown to be true for an inhomogeneous medium between two receivers in Gouédard et al (2008). The assumption for retrieving the Green's function is that noise sources are distributed over all azimuths, a condition which has been shown to be satisfied for southern 


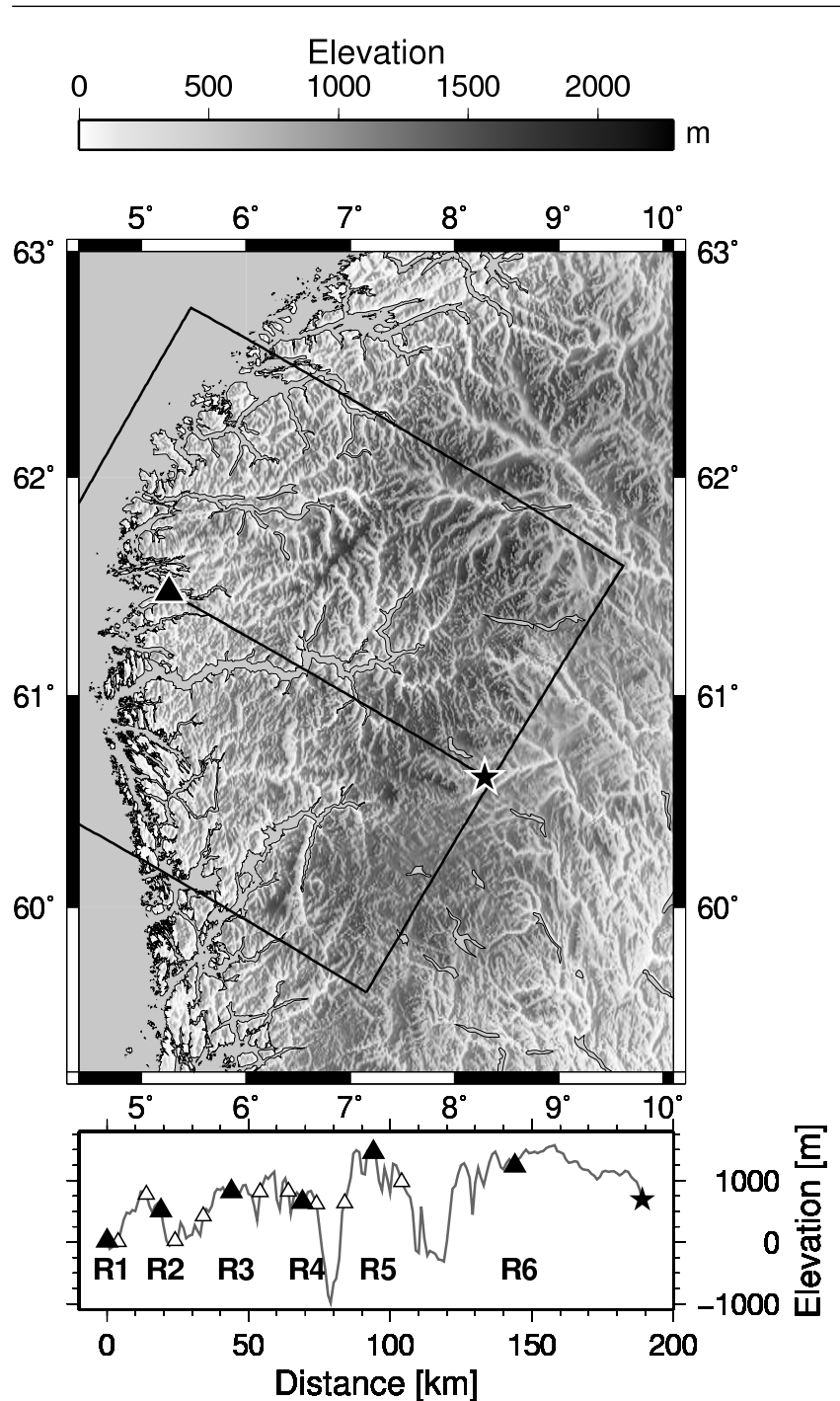

Fig. 1 Top: Topographic map of western Norway outlining the limits of the model for simulation. The box is centered around the line between source (star) and receiver R1 (triangle). Bottom: Topographic profile of the analyzed section. Filled triangles indicate receivers discussed in the text and open triangles additional data points used in Figure 5 b. Star depicts source location in simulation.

Norway (Köhler et al, 2011). In order to simulate how the topography affects noise studies, it is therefore sufficient to analyze how it affects Green's functions between receivers.

We use the spectral element method (Komatitsch and Vilotte, 1998; Peter et al, 2011) to generate synthetic seismograms. For the numerical simulation of three-dimensional seismic wave propagation, we choose a homogeneous medium $\left(V_{s}=3.4 \mathrm{~km} / \mathrm{s}, V_{p}=6 \mathrm{~km} / \mathrm{s}, \rho=2.6 \mathrm{~g} / \mathrm{ccm}\right)$ with a free surface on top and absorbing boundary conditions elsewhere. The horizontal dimension of the model is quadratic with edges of $250 \mathrm{~km}$ length and a depth of $40 \mathrm{~km}$. The model is meshed into elements of about $1 \mathrm{~km}^{3}$ volume. A deformed mesh is used whose layers are following the topography along the free surface. The profile that we chose to analyze is the interstation path between stations NWG16 (source) and NWG14 (receiver R1) of the MAGNUS network. An explosion at the surface with a ramp source time function ( 1 second duration) is chosen for the point source. The generation of a proper Green's function would require using a single force as point source. An explosion was used because simulations with point forces generated seismograms with dominant energy at too high frequencies to enable a proper dispersion analysis. Since we will only compare the velocities measured with and without topography, the choice of source type will not affect the results.

First, we generated a reference data set without topography. Then, the real observed 3-D topography within the same $250 \mathrm{~km} \times 250 \mathrm{~km}$ box centered around the interstation profile is included. For this 3-D simulation, the bathymetry of the fjords is considered as a free surface and no water is included. We studied the effect of the deep water bodies in an additional 2-D simulation which showed energy loss due to acoustic waves trapped in the fjord. However, apart from damping, the main wave front travels along the fjord bottom and is only minorly disturbed.

In order to quantify the effect of stronger topographic contrasts than those observed in southern Norway, we also generate synthetic data for a model where we scaled the real topography by a factor of two (referred to as 'scaled' topography in the following). Simulations are conducted with a sampling rate of $200 \mathrm{~Hz}$ and a duration of 120 seconds. Resolvable periods are between about 0.25 and 10 seconds.

\section{Results of simulation}

Figure 2 shows the waveforms (displacement) from the three different models, measured at four distances from the source. The impact of the topography on the arrival time of the main Rayleigh wave front is in general only minimal. The wave appears to be slightly late with the scaled topography at long distances but this apparent delay could be an effect of changing frequency content. At the most remote location R1, the amplitude in the tail of the Rayleigh wave increases with scaled topography, probably due to enhanced scattering and interference. At the other locations, the unscaled topography does not affect the wave amplitude of the main Rayleigh wave significantly. Only when the topography is exaggerated, a marked decrease in amplitude relates to scattering of energy. Similarly, coda waves are much more prominent when topography is scaled, in which case two wavetrains related to reflections from deep fjords are clearly visible at the two first locations (R5 and R6). Note that these reflections do not present a simple moveout in our distance plots due to the complex 3-D geometry of the fjords offline from the profile. Higher frequent scattered waves arrive not only later in the coda but also before the main onset of the direct 

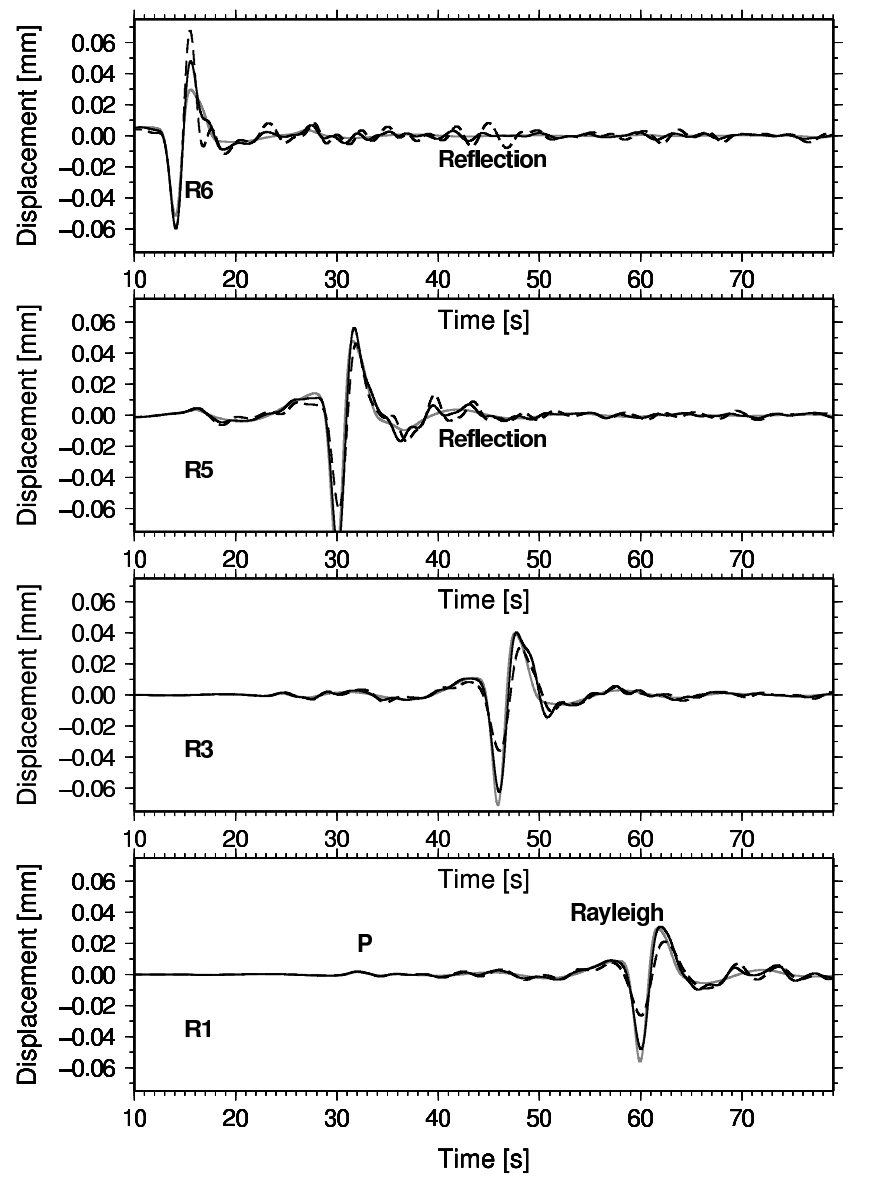

Fig. 2 Synthetic waveforms: Vertical component measured at four different distances from source (see Figure 1). Data obtained from a model without topography (grey line), with real topography (black line), and real topography scaled by a factor of two (black dashed line) are shown. Names of observed phases: direct P-wave, Rayleigh wave, and reflection from fjord.

Rayleigh wave due to conversion between body and surface waves (Rodgers et al, 2010).

We apply time-frequency analysis (Levshin et al, 1989; Ritzwoller and Levshin, 1998) to the vertical displacement of the synthetic wavefield to measure Rayleigh wave phase velocities. Due to attenuation and the limits of the model, only surface wave from 3 to 10 second period are analyzed. Figure 3 shows the results after correction for the source phase (explosion) and $2 \pi$ phase ambiguity. The theoretical phase velocity value in a homogeneous halfspace is 3.134 $\mathrm{km} / \mathrm{s}$ and is correctly measured at the shortest periods in the flat model. However, measured velocities slightly increase at longer periods due to the vertical limitation of the model. This becomes more evident by comparing measured phase velocities from the $40 \mathrm{~km}$ deep model with measurements from a simulation with a $30 \mathrm{~km}$ deep model (red symbols in Figure 3). The latter shows stronger dispersion towards longer periods.

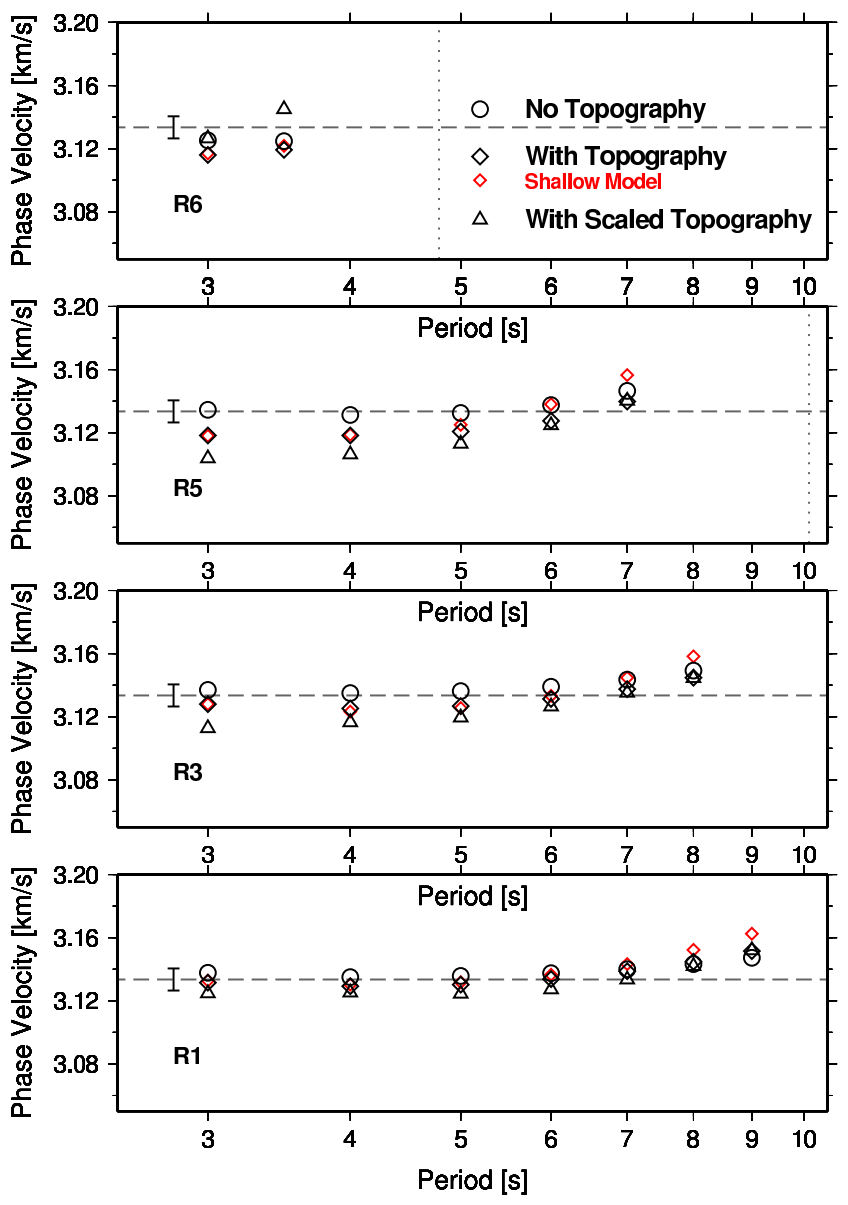

Fig. 3 Phase velocity measured at four different locations for different models. Dashed horizontal line shows theoretical phase velocity of Rayleigh waves (homogeneous halve-space). Vertical dotted lines indicate period obtained from upper wavelength limit (one third of distance between source and receiver). Error bars on left handsides represent average measurement uncertainty obtained for real data (Köhler et al, 2011).

As expected from Figure 2, the effect of topography is minimal for the unscaled profile. For receivers R1 and R3 it lies within the measurement uncertainties of real data from southern Norway. Nevertheless, one can observe a tendency to underestimate phase velocities, in particular for the scaled profile, and we should note that all velocity anomalies are negative, in accordance with the predictions made by Huang and Maradudin (1987). This bias increases with the amplitude of the topography and decreases with increasing period. It is largest at location R3 and R5 due to locally large topographic contrasts in the vicinity of the receivers. For realistic topography in southern Norway the measured bias in phase velocity is, however, not larger than about $-0.5 \%$ for the receivers considered here (R5) or $-0.65 \%$ for the assessed part of the profile (see R4 in Figure 5a) at 3 seconds period. For longer distances $(>140 \mathrm{~km})$ and longer periods the impact is clearly smaller and below significance. 


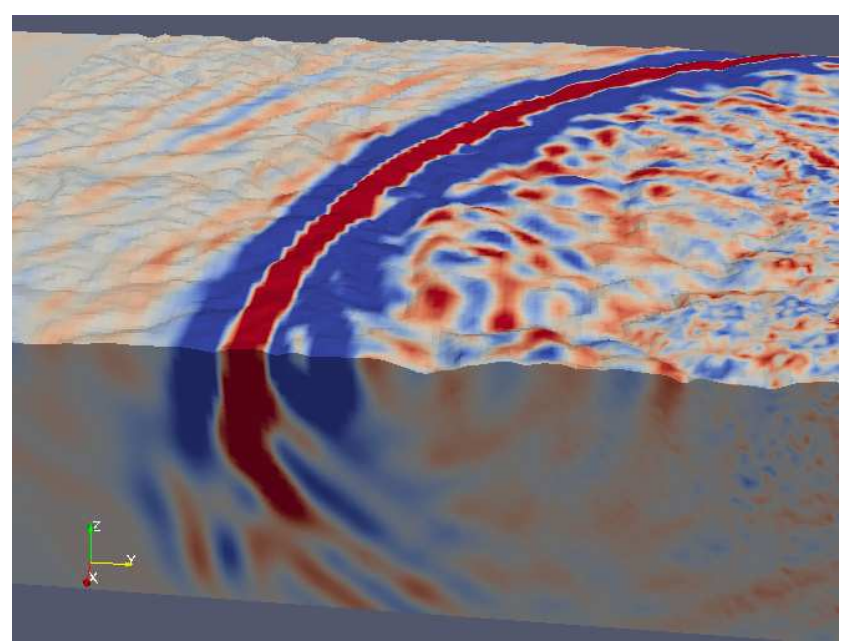

Fig. 4 Snapshoot of synthetic wavefield (vertical component). Model is cut along the chosen profile. Waveform with largest amplitude is the main Rayleigh wave front.

Figure 4 shows details on how the wavefield is scattered due to the topography. In particular, it is clearly visible that waves are also back-scattered. The main wave front itself, however, is not much disturbed. The depth of the model in Figure 4 is $40 \mathrm{~km}$ and we can see that the energy of the Rayleigh wave in the ambient noise frequency range penetrates down to about $20 \mathrm{~km}$. Rayleigh waves at these short periods are, as a rule of thumb, mostly sensitive to depths (in $\mathrm{km}$ ) between 0.5 and 2 times the value of the period, hence down to approx. $20 \mathrm{~km}$ for 10 seconds period. Since the variations in topography are on the order of $\leq 3 \mathrm{~km}$ it is thus not surprising that the topography scatters part of the energy but is not able to significantly affect the overall velocity of the wavetrain. Measurable effects are hence only present at periods below 6 seconds.

\section{Generalization of maximum bias}

\subsection{Southern Norway}

From these simulations it is clear that the amplitude of the topography, as well as its spectral distribution affects Rayleigh wave phase velocity only weakly. As introduced earlier, a simple model to assess the effect of topography is to use the surface distance following the topography when calculating the phase velocity instead of the "straight line", horizontal distance.

Rayleigh waves are unlikely to follow topographic variations at wavelengths smaller than their own wavelength, and one can speculate that, for each Rayleigh wavelength $\lambda_{R}$, there exists a corner-wavelength $\lambda_{t}$ of topography below which topographic variations will not affect the measured velocity of the Rayleigh wave, hence $\lambda_{t}=\alpha \times \lambda_{R}$, with $\alpha$ being a factor to be determined in the following. The sur- face distance $d_{\lambda_{t}}$, and thus the difference to the horizontal distance $\delta d=d_{\infty}-d_{\lambda_{t}}$, will decrease when topography is low-pass filtered at increasing corner-wavelengths. Our hypothesis is that the bias due to topography can be estimated by calculating the surface distance after low-pass filtering the topography at the appropriate corner-wavelength. This velocity bias would follow the simple relation:

$\frac{\delta c}{c_{0}}=\frac{\delta d}{d_{\lambda_{t}}}$

where $\delta c=c-c_{0}, c$ is the measured (biased) phase velocity and $c_{0}$ is the unbiased phase velocity.

We test this hypothesis with the synthetic data and evaluate how $\alpha$ (and the appropriate corner-wavelength $\lambda_{t}$ ) can be found. Since there is apparently no effect for periods $\geq$ 6 seconds, we present in Figure 5 the biases in phase velocities at 3, 4, and 5 seconds period measured at stations R1 to $\mathrm{R} 5$ both with unscaled and scaled topographies as function of $\delta d / d_{\lambda_{t}}$. We use $\alpha=2.5$. The reason for our choice is discussed later.

If our hypothesis is valid and an appropriate corner-wavelength $\lambda_{t}$ is used, we should observe a linear relationship according to Equation (1) (grey line in Figure 5a). It is clear from Figure 5a that this is not the case although there is a clear trend of larger bias with increasing difference in distance. The spread is, however, too large to explain our observations by increasing surface distance alone.

Hence, other effects have to be taken into account. It can be expected that scattering and interference due to locally large topographic contrasts in our 3-D model leads to biases in measured velocity. Multiple scattering has been shown to slightly reduce the apparent phase velocity of Rayleigh and Love waves in models with random heterogeneities (Maupin, 2002) and it is possible that this phenomenon occurs here as well. Furthermore, wavefront healing past the main mountain range could compensate this effect for receivers in "flatter" terrain.

If there is no simple relation between phase velocity error and distance, the observed effects may have a local origin. Therefore, we compute the average gradient $G$ of the filtered topography within an area of $15 \mathrm{~km}$ radius around each receiver and color-code the data points in Figure $5 \mathrm{a}$ accordingly. Results show that data measured at receivers with relatively flat close-by topography (below 14\% slope) follow somewhat our predicted linear relationship, particularly in the scaled topography model. Phase velocity errors from receivers with strong topographic contrasts in their vicinity, i.e. stations close to fjords such as R4 and R5 are, however, clearly underestimated. Moreover, the errors for the receivers in the real topography (dashed box) are poorly predicted by our hypothesis of increased travel distance of seismic waves. Nevertheless, if we fit a line to the datapoints for a range of values for $\alpha$ (only receivers with close-by slopes 
a) Velocity Bias vs. Distance

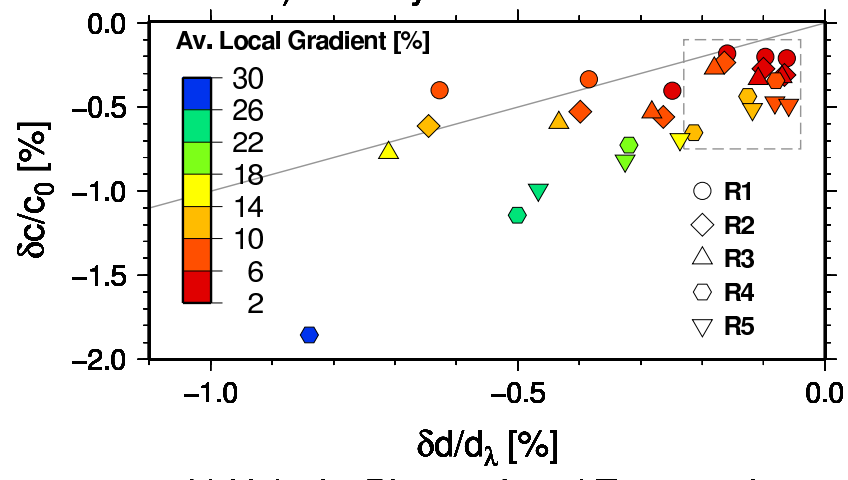

b) Velocity Bias vs. Local Topography

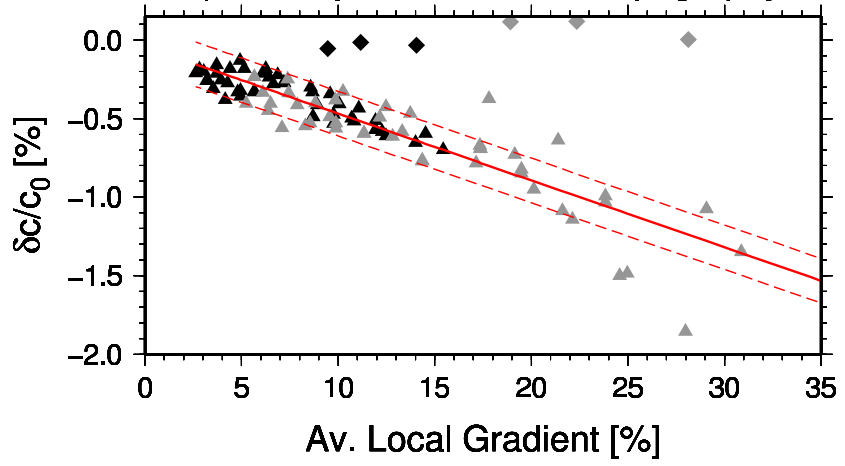

Fig. 5 Measured velocity bias versus topography. a) Grey line indicates our hypothesis based on Equation (1). Data is compiled from 5 different receivers (R1 to R5) at 3, 4, and 5 seconds period, using both observed (inside dashed box) and scaled topography (outside box). Color code is local average topography gradient in percent within an area of $15 \mathrm{~km}$ radius around receiver. Measured values from flat topography simulation are used for $\mathrm{c}_{0}$. b) Measured velocity bias as a function of local gradient within $15 \mathrm{~km}$ radius and linear regression (red line, standard deviation dashed). Measurements from additional receivers are added (see Figure 1, without R6). Black symbols correspond to real and grey to scaled topography. Diamonds indicate data for station located at $\mathrm{km} 84$ which are not used for regression.

$\leq 14 \%$ ) through weighted linear regression, we find that a corner wavelength factor of $\alpha=2.5$ yields a slope closest to 1 . The hypothesis may thus explain the order of the observed velocity bias only under the assumption of relatively smooth local topography and large surface distance differences $\left(\delta d / d_{\lambda_{t}}<-0.15 \%\right)$ and is therefore not suitable to predict the maximum expected error in the general case in seismological practice.

Figure $5 \mathrm{~b}$ shows the velocity bias as a function of local gradient computed from the corner-wavelength-filtered topography $\left(\lambda_{t}=\alpha \times \lambda_{R}\right)$ within an area of $15 \mathrm{~km}$ radius (same as done for color-code in Figure 5a). Measurements from additional receivers are added (see Figure 1, without R6). There seems to be a linear relationship between local topography and the phase velocity error. The obvious outliers with almost no bias belong to the receiver at $\mathrm{km} 84$ on the profile (diamonds in Figure 5b). The location of this receiver is exceptional, positioned on a long steep slope of about $27 \%$ which is not a realistic site for a seismic station, and it is unclear why the observed bias is so low. If we omit this particular receiver we obtain the following linear regression:

$\frac{\delta c}{c_{0}}[\%]=-0.0425 \cdot G[\%]-0.044$,

with a prediction error of $0.141 \%$. We tested different factors $\alpha$ to determine the corner-wavelength for the topography filter, and various radii for the area of average gradient and found that $\alpha=2.5$ and a radius of $15 \mathrm{~km}$ gives the lowest regression error. Clearly, there is a trade-off in the determination of these two parameters but results vary only minorly with small variations in $\alpha$ and radius and, more importantly, the values are physically plausible. Rayleigh waves with 3-5 seconds period have a wavelength of about 9-16 $\mathrm{km}$ and (from our analysis) sense wavelengths of topography $\geq 23-40 \mathrm{~km}$ or local relief within a region of around 1-2 wavelengths radius from the receiver.

It is also noteworthy that, contrary to the distance hypothesis, the data points from the unscaled topographic model fit the observed linear relation well and even slightly better (prediction error of $0.069 \%$ ) than the data from the scaled topography, suggesting that the spread increases with larger topographic contrasts. We will show later that the local gradients from the scaled topographic model are on a global basis rather unrealistic. It seems thus that the phase velocity error of shortest period surface waves from 3-D topography may be assessed by this simple estimate although a complete validation of the presented observations would certainly require a more rigorous analysis than we have done here.

It is difficult to give a physical explanation for this striking linear relation between velocity bias and local topographic gradient. As mentioned above, multiple scattering and wavefront healing could lead to such localized effects. Larger topographic contrasts certainly increase the degree of scattering and thus likely increase the bias in measured phase velocity. One can speculate that this could lead to a relation which is in first order linear, however a simple physical explanation cannot be given.

We have also compared our phase velocity shifts with the analytical results of Huang and Maradudin (1987). They model the phase velocity shift for profiles with random topographic variations having a Gaussian spectrum. The power spectrum of the topography of Norway is not Gaussian but follows basically the power law in $k^{-2}$ (see Figure $7 \mathrm{~b}$ ) found to fit the Earth's topography in very many settings (Turcotte, 1997). Although the best-fitting Gaussian power spectrum with a correlation length of $50 \mathrm{~km}$ and an amplitude of $2.25 \mathrm{~km}^{2}$ fits the long-wavelength part of the spectrum quite well, it clearly underestimates the amount of topography at smaller wavelengths. Huang and Maradudin's method predicts at $10 \mathrm{~km}$ wavelength a very small phase velocity shift of $-5 \times 10^{-4} \%$ for Norway and a shift 4 times larger for the 

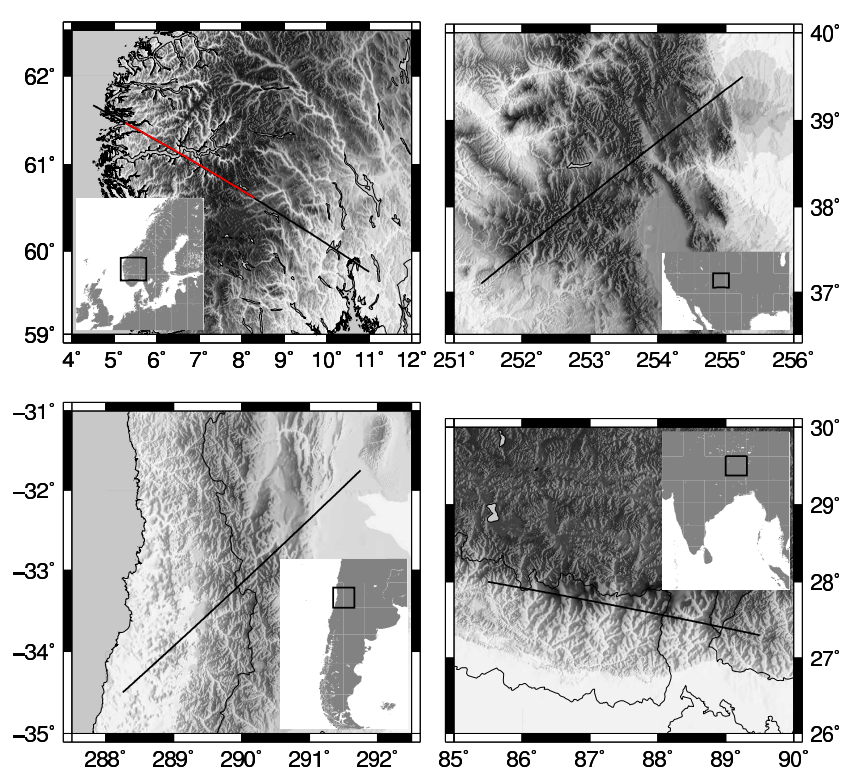

Fig. 6 Profiles through different mountains ranges: Southern Norway (Scandes), Rocky Mountains, Andes, Himalayas. Short profile from Figure 1 is shown as a red line for southern Norway.

scaled topography. This is clearly much smaller than what we measure and may be related to the fact that the Gaussian power law is not appropriate in our case. Palasantzas (1994) has shown that the amplitude of surface scattering phenomena depends strongly on the fractal dimension of the surface involved and increases from Gaussian-type surfaces to small-wavelength richer surfaces as we have.

\subsection{Other mountain ranges}

Although smaller in lateral extension and at a lower average altitude than other major mountain ranges on Earth, the Scandes of southern Norway have a roughness in topography that is not dislike the ones of major ranges. In order to evaluate whether our simulations can be used to estimate the bias related to topography elsewhere on Earth, we choose to compare the profile used in our simulations to three profiles in other main mountain ranges: the southern Rocky Mountains, the Andes, and the Himalayas (Figure 6). The profile for the latter region is a rather extreme case oriented parallel to the mountain chain across major valleys, within the area of the highest elevations worldwide (Mt. Everest region). Ambient noise tomography has been carried out in that region by Guo et al (2009). The southern Rocky Mountains have been recently sampled by the USArray (Lin et al, 2009).

The topographic profiles are shown in Figure 7a. The Himalayas stands as having a rougher topography than the other ranges, but the altitude variations are not much larger than in the scaled version of the Scandes topography used in our simulations. This is more clear in the spectra of the pro- files (Figure 7b) where we see that the amplitude of topography at long wavelengths is similar in the Himalayas and in the scaled Scandes, whereas the unscaled Scandes compares well with the Rocky Mountains and the Andes.

If the distance hypothesis was valid, this similarity would persist in the prediction of velocity error following Equation (1), for which we use sections of all profiles of the same length as the profile source-R1 in our simulations for southern Norway (Figure 7c). In this case, the effect in southern Norway would be of the same order of magnitude as in the Andes and larger than in the Rocky Mountains while the bias predicted for the Himalayas would be largest and of the same order as predicted for the scaled profile of southern Norway.

However, if we predict the maximum bias from local topographic effects (following Equation (2)) at the location of the largest topographic gradient in each profile, the predictions are quite different. First, the maximum expected error is significantly larger than for the distance-based prediction, and secondly, the mountain ranges compare differently. The predicted bias for the unscaled profile in Norway is now of the same order as the Himalayas and can be as large as $0.7 \%$ at $9 \mathrm{~km}$ wavelength while the maximum error for the profiles of the Rocky Mountains and the Andes is smaller. Since the Himalayan profile represents a sort of maximum effect we may obtain globally (for most of the Himalayas the bias is probably smaller), the slopes computed from the scaled Norwegian profile are obviously unrealistic for global observed topography.

In general, the biases decrease towards longer periods but increase when going to higher frequencies. We cannot exclude that the distance hypothesis will gain importance at periods below 3 seconds and thus a rapid increase of the velocity bias at wavelengths below 6-8 km. However, recent modeling has shown that the apparent bias will likely be smaller due generation of $\mathrm{Rg}$ precursors from conversion of P-to-Rg waves at periods $\leq 2$ seconds (Rodgers et al, 2010). Since typical phase velocity anomalies obtained from seismic noise tomographies in different regions worldwide are of the order of $2-3 \%$ and larger, we can conclude that topography will not have a significant effect, given that strong local effects at the receiver are absent at short wavelengths.

\section{Conclusions}

We have investigated the effect of topography on phase velocity measurements of short period surface waves, relevant for seismic tomography based on ambient seismic noise. By means of synthetic data we found that the bias due to topography on phase velocity measurements is always negative and may be relevant for periods $\leq 5$ seconds. For southern Norway we measured a maximum underestimation of phase 


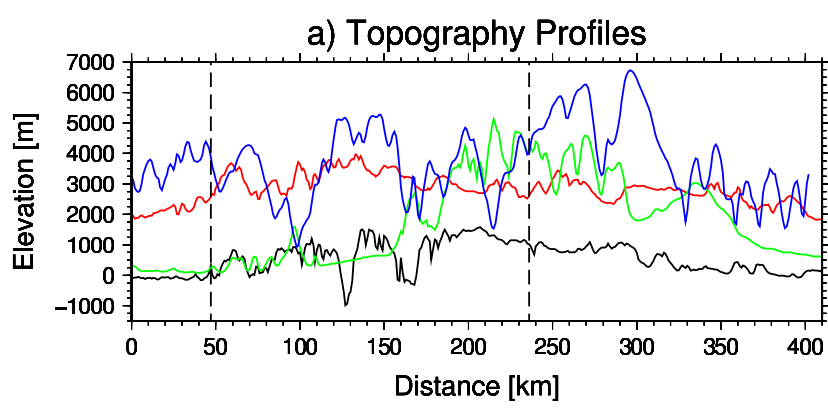

b) Topography Spectra

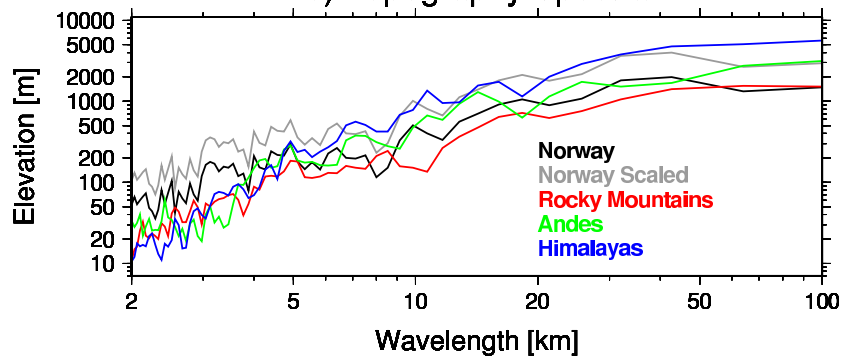

c) Predicted Velocity Bias due to Topography

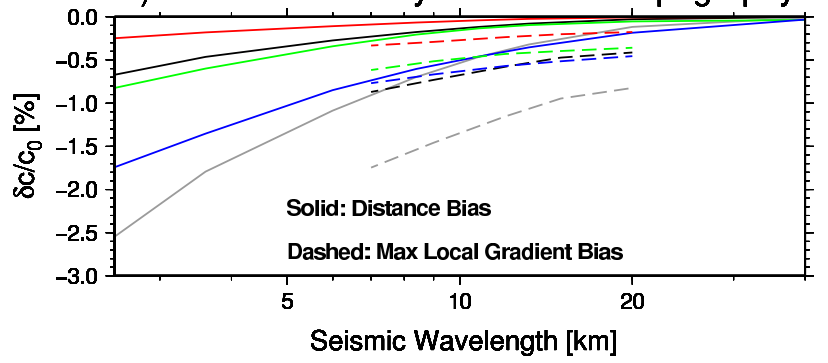

Fig. 7 Topographic profiles, spectra and predicted phase velocity bias on profiles in Figure 6. a) Elevation along profiles. Vertical dotted lines delimit segments used in c) (Source to receiver R1 in case of Norway). b) Wavelength spectra of (entire) profiles in a). c) Solid lines: Predicted velocity bias on segment of profiles indicated in a) based on distance hypothesis. Dashed lines: Maximum predicted velocity bias due to local topography effects. Velocity bias is plotted against seismic wavelength obtained from corner wavelength by dividing through 2.5 .

velocities of about $-0.65 \%$ on realistic interstation paths. However, synthetic data showed that this effect may theoretically double if topographic contrasts stronger than the observed ones exist in the vicinity of a receiver. Nevertheless, for longer periods and longer source-receiver lines locally large contrasts are "averaged" out by 3-D effects, and the impact of realistic topography on phase velocity measurements was found to be below significance.

We have shown that the observed velocity bias cannot be explained by increased surface distance along topography alone, but is related to the presence of locally large topographic contrasts. We found a linear relation between phase velocity error and the locally averaged, topographic gradient. Since the physical reason for that is unclear, further investigations of this observation are required.

By comparison of topographic profiles from mountain ranges worldwide, we propose that our results in southern
Norway are also representative for other regions and estimate that the maximum effect of topography on phase velocity measurements does not exceed $-0.7 \%$ globally.

Acknowledgements Simulations have been conducted on the TITAN cluster of the University of Oslo. This work has been done in the framework of the ESF EUROCORES TOPO-EUROPE Program 07TOPO-EUROPE-FP-014. We acknowledge financial support from the Research Council of Norway. Figures have been prepared using the Generic Mapping tools (Wessel and Smith, 1998). Special thanks go to Michel Heeremans for providing the bathymetry data of southern Norway, Hom Nath Gharti for introducing into the spectral element code, and to Dani Schmid for access to the 3-D meshing program. We appreciate the comments and suggestions of two anonymous reviewers which helped to improve this manuscript.

\section{References}

Eguiluz AG, Maradudin AA (1983) Frequency shift and attenuation length of a rayleigh wave due to surface roughness. Phys Rev B 28(2):728-747, DOI 10.1103/PhysRevB.28.728

Fu L, Wu R (2001) A hybrid BE-GS method for modeling regional wave propagation. Pure appl geophys 158(7):1251-1277, DOI 10.1007/PL00001222

Fu L, Wu R, Campillo M (2002) Energy partition and attenuation of regional phases by random free surface. Bull Seism Soc Am 92(5):1992-2007, DOI 10.1785/0120000292

GLOBETaskTeam, Hastings D, Dunbar P, Elphingstone G, Bootz M, Murakami H, Maruyama H, Masaharu H, Holland P, Payne J, Bryant N, Logan MJ TL and, G S, MacDonald J (1999) The Global Land One-kilometer Base Elevation (GLOBE) Digital Elevation Model, Version 1.0. National Oceanic and Atmospheric Administration, National Geophysical Data Center, 325 Broadway, Boulder, Colorado 80305-3328, USA, Digital data base on the World Wide Web (URL: http://wwwngdenoaagov/mgg/topo/globehtml) and CDROMs

Gouédard P, Stehly L, Brenguier F, Campillo M, Colin de Verdière Y, Larose E, Margerin L, Roux P, SánchezSesma F, Shapiro N, Weaver RL (2008) Cross-correlation of random fields: mathematical approach and applications. Geophysical Prospecting 56:375-394, DOI 10.1111/j.1365-2478.2007.00684.x

Guo Z, Gao X, Yao H, Li J, Wang W (2009) Midcrustal low-velocity layer beneath the central Himalaya and southern Tibet revealed by ambient noise array tomography. Geochem Geophys Geosyst 10:Q05,007, DOI 10.1029/2009GC002458

Huang X, Maradudin AA (1987) Propagation of surface acoustic waves across random gratings. Phys Rev B 36(15):7827-7839, DOI 10.1103/PhysRevB.36.7827 
Köhler A, Weidle C, Maupin V (2011) Directionality analysis and Rayleigh wave tomography of ambient seismic noise in southern Norway. Geophysical Journal International 184:287-300, DOI 10.1111/j.1365246X.2010.04830.x

Komatitsch D, Vilotte J (1998) The spectral element method: an efficient tool to simulate the seismic response of 2D and 3D geological structures. Bull Seism Soc Am 88(2):368-392

Levshin A, Yanovskaya T, Lander A, Bukchin B, Barmin M, Ratnikova L, Its E (1989) Seismic surface waves in a laterally inhomogeneous earth. ed. Keilis-Borok, V.I., Kluwer, Norwell, Mass.

Lidmar-Bergström K, Ollier C, Sulebak J (2000) Landforms and uplift history of southern Norway. Global and Planetary Change 24(3-4):211-231, DOI 10.1016/S09218181(00)00009-6

Lin F, Ritzwoller M, Snieder R (2009) Eikonal tomography: surface wave tomography by phase front tracking across a regional broad-band seismic array. Geophysical Journal International 177(3):1091-1110, DOI 10.1111/j.1365-246X.2009.04105.x

Maradudin A, Huang X, Mayer A (1991) Propagation of shear horizontal surface acoustic waves parallel to the grooves of a random grating. Journal of Applied Physics 70(1):53-62, DOI 10.1063/1.350270

Maupin V (2002) The amplitude of the love-rayleigh discrepancy created by small-scale heterogeneities. Geophysical Journal International 150(1):58-64, DOI 10.1046/j.1365-246X.2002.01676.x

Mayer A, Zierau W, Maradudin A (1991) Surface acoustic waves propagating along the grooves of a periodic grating. Journal of Applied Physics 69(4):1942-1947, DOI $10.1063 / 1.348969$

Palasantzas G (1994) Eigenwave spectrum of surface acoustic waves on a rough self-affine fractal surface. Phys Rev B 50(24):18,670-18,673, DOI 10.1103/PhysRevB.50.18670

Peter D, Komatitsch D, Luo Y, Martin R, Le Goff N, Casarotti E, Le Loher P, Magnoni F, Liu Q, Blitz C, Nissen-Meyer T, Basini P, Tromp J (2011) Forward and adjoint simulations of seismic wave propagation on fully unstructured hexahedral meshes. Geophysical Journal International 186, DOI 10.1111/j.1365-246X.2011.05044.x

Ritzwoller M, Levshin A (1998) Eurasian surface wave tomography: Group velocities. J Geophys Res 103(B3):4839-4878, DOI 10.1029/97JB02622

Rodgers A, Petersson N, Sjogreen B (2010) Simulation of topographic effects on seismic waves from shallow explosions near the north korean nuclear test site with emphasis on shear wave generation. J Geophys Res 115:B11,309, DOI 10.1029/2010JB007707
Sabra K, Gerstoft P, Roux P, Kuperman W, Fehler M (2005) Extracting time-domain Green's function estimates from ambient seismic noise. Geophys Res Lett 32:L03,310, DOI :10.1029/2004GL021862

Shapiro N, Campillo M (2004) Emergence of broadband Rayleigh waves from correlations of the ambient seismic noise. Geophys Res Lett 31:L07,614, DOI :10.1029/2004GL019491

Snieder R (1986) The influence of topography on the propagation and scattering of surface waves. Physics of the Earth and Planetary Interiors 44(3):226-241, DOI 10.1016/0031-9201(86)90072-5

Stratford W, Thybo H, Faleide J, Olesen O, Tryggvason A (2009) New Moho Map for onshore southern Norway. Geophysical Journal International 178:1755-1765, DOI 10.1111/j.1365-246X.2009.04240.x

Turcotte D (1997) Fractals and chaos in geology and geophysics. Cambridge University Press

Weidle C, Maupin V, Ritter J, Kvaerna T, Schweitzer J, Balling N, Thybo H, Faleide J, Wenzel F (2010) MAGNUS-A seismological broadband experiment to resolve crustal and upper mantle structure beneath the southern Scandes mountains in Norway. Seismological Research Letters 81(1):76-84, DOI 10.1785/gssrl.81.1.76 Wessel P, Smith W (1998) New, improved version of GMT released. EOS Trans Amer Geophys Un 79(47):579-579

Wu X, Wu R (2001) Lg-wave simulation in heterogeneous crusts with surface topography using screen propagators. Geophysical Journal International 146(3):670-678, DOI 10.1046/j.1365-246X.2001.00489.x 